\title{
Talking to the Conversation Machine: An Empirical Study
}

\author{
Catherine G. Wolf*, Max Kassler, Wlodek Zadrozny, Lukasz Opyrchal \\ IBM Thomas J. Watson Research Center \\ 30 Saw Mill River Road, Hawthorne, NY 10532 USA \\ *cwolf@watson.ibm.com
}

\begin{abstract}
This paper describes an empirical study of a prototype conversational speech system. the Conversation Machine. The system allowed users to carry out a number of common banking transactions using a conversational-style interface over the telephone. The study reports detailed findings on how users interacted with the Conversation Machine. categories of error. measures of accuracy and goal success. subjective reaction to the Conversation Machine, and correlations between objective measures and important measures of user satisfaction. Among the findings of interest are that users preferred the speech system over a telephone keypad system and that goal success was more highly correlated with measures of user satisfaction than was recognition accuracy. Implications for the design of conversational user interfaces based on an analysis of errors are also discussed.
\end{abstract}

KEY WORDS speech recognition. speech interface, natural language

\section{INTRODUCTION}

Jenny is about to board the plane for a two-week vacation. A thought suddenly flashes through her mind: She forgot to pay her Mastercard bill! She dashes for the nearest phone. dials the number for her automated speech banking system and says, "Please pay my Mastercard bill." Her request is recognized and the system voice responds. "Your Mastercard balance is $\$ 2005.56$. You do not have enough money in your checking account to pay this bill. Would you like me to transfer money from your savings account and pay the bill?" Jenny responds. "Yes." and the system gives a message confirming payment of the bill. Jenny boards the plane. relieved that all is in order.

When typical consumers think of using speech to interact with a computer. they usually imagine a conversational interaction in which the computer recognizes not only their words. but understands their intentions as well. The possibility of carrying on an unconstrained conversation with a computer in which the computer understands its partner in a completely human-like way is not likely to be realized in the foreseeable future. However. interactions such as the one illustrated by the scenario above are likely to become a reality in the next few years. As suggested by Danis and Karat (1995), researchers can accelerate the development of usable speech interfaces and the underlying speech technology by picking applications whose requirements are a good match to the strengths and limitations of the technology.

This paper describes an empirical study of a prototype conversational speech system. the Conversation Machine. The domain of application was home banking. The system allows users to carry out a number of common banking transactions using a conversational-style interface over the telephone. The study had several purposes: 
-To understand how people interact with the system in order to improve the usability of the system. For example, we wanted to know what strategies people used when the system made an error.

- To collect data about the way people spoke to the system in order to more adequately cover the variety of utterances people use.

-To assess the system 's accuracy in recognizing users" utterances.

- To assess users success in accomplishing their goals with the system, and their reactions to the system.

This work benefits from the earlier research of Yankelovich, Levow and Marx (1995), Marx and Schmandt (1996). Halstead-Nussloch (1989), and Clark and Brennan (1991) on the design of conversational interfaces, and the work of Hansen, Novick and Sutton (1996) on the design of effective speech prompts. The study presented here contributes to the field by reporting detailed empirical findings on how users interacted with the Conversation Machine. categories of error, measures of accuracy and goal success, subjective reaction to the Conversation Machine, and correlations between objective measures and important measures of user satisfaction. The paper also discusses the implications of these findings for the design of conversational speech interfaces.

A conversational speech interface offers a number of potential advantages over keypad input for telephonebased services such as the current banking application:

-A conversational interface allows users to make requests in their own words. In a keypad interface, the burden is on the users to figure out which of the system options best matches their goals.

-A conversational interface may have a flat, nonhierarchical command structure which allows users to accomplish their goals directly (e.g., "What is my checking balance?"). Keypad interfaces require the users to learn and navigate several levels of a hierarchical menu structure to accomplish their goals.

-A conversational speech interface allows users to make compound requests (e.g. "Pay my phone and electric bills."). Keypad interfaces typically require users to accomplish their goals as a series of discrete tasks.

While a conversational speech interface promises considerable benefits for an application such as banking over the telephone, there are also a number of challenges in the design of such systems. Due to the imprecise nature of speech recognition. there may be a mismatch between the user's utterance and what is recognized by the system. Since speech recognizers often fail in non-human ways. the user may be surprised by the result (Rhyne and Wolf. 1993). Furthermore, the user may have little idea how to recover from a recognition error. In addition. the appearance of some human-like language ability may make it difficult for users to distinguish what the system can and cannot do. Such issues as these cannot be solved by improvements to the speech and natural language technology alone, but must also be addressed by the design of the user interface.

\section{THE SYSTEM}

\subsection{User Interface Design and Application Functionality}

The system allowed users to do the following operations: get checking and savings account balances, transfer money between checking and savings accounts. get the balance for four bills, pay these bills, summarize the bills, summarize all bills and accounts. get help, and cancel a transaction.

The system was capable of some compound actions. such as paying several bills at once, and understood some forms of deictic reference, such as pronouns.

The command structure was flat. and the top-level prompt. "What else can I do for you?" simply signaled that the system was ready to accept another request. Some dialogs required additional information from the user for completion. In these sub-dialogs, the users were asked for the additional information and only responses appropriate to the dialog were accepted. For operations which changed the balance of an account. the user was asked to confirm the transaction before it was executed

For greater accuracy, dollar amounts were entered using the keypad. Even if users spoke the amount in a request, they were prompted to enter it using the keypad.

When a recognition failure occurred at a top-level dialog, the user was asked to rephrase the request. When an acceptable response was not given within a sub-dialog. several levels of prompting were used, giving progressively more explicit instructions. 
The prompts consisted of pre-recorded human speech by a female speaker. The prompts used a polite. firstperson style.

\subsection{System Components}

The Conversation Machine consists of three major modules: Natural Language/Dialog Machine, Computer Telephony System, and Continuous Speech Recognition. The modules communicate through TCP/IP. The system is modular.

The Conversation Machine ran on an $\mathrm{RS} / 6000$ model 370 under AIX.

Natural Language -- The natural language grammar is represented as a collection of constructions (Goldberg. 1995), i.e. data structures where syntactic. semantic and pragmatic information are combined in one record. This representation has important computational advantages of being general. compact and object oriented (Zadrozny. 1996).

Dialog Machine --The Dialog Machine works in the conversation-for-action paradigm, i.e. it tries to find out which action to perform and what the parameters of the action are. In other words. it is a finite collection of states and transitions plus a small number $(<20)$ of global, contextual variables that help determine the next state and the utterance interpretation. If the whole utterance cannot be interpreted within a given context. the largest subpart that makes sense is tried. Because of the possible ambiguities, there is a small set of rules ( 5 clauses) expressing the preferences in interpretation (cf. Zadrozny, 1995).

Speech Recognition -- The system as tested used the BBN Hark Continuous Speech Recognition System. The IBM Continuous Speech Recognition System. has more recently been integrated into the Conversation Machine. Both recognizers support large vocabulary. and both work best if at any point of a conversation the active vocabulary does not exceed a few hundred words.

Computer Telephony System - The IBM DT/6000. version 1.6 system was used.

\section{THE STUDY}

\subsection{Tasks and Procedure}

Since one of the goals of the study was to collect information on how people spoke to the system. we did not want to "put words in their mouths" by presenting the tasks they were to do in words they might use to accomplish these tasks. Accordingly, we created scenarios that either described a situation the study participants were to resolve or required the participants to fill in missing information in a table, without using phrases they might employ to accomplish the goal. An example of the first kind of scenario is: "You are at the airport leaving for a twoweek vacation. You have an uneasy feeling that you forgot the electricity bill. Check to see if it needs attention, and if so. take care of it."

Eight scenarios were used. with the intention that these would give participants an opportunity to use all of the functions of the system. The scenarios were given to the participants in a paper booklet. The first two scenarios were designed to be relatively simple, in order to allow participants to become familiar with the experimental procedure.

Participants were given verbal and written instructions about the procedure. including an example task. They were told they would be interacting with a computerized voice recognition system and to "make requests as if you were speaking to a human."

Participants spoke to the system using a telephone handset over a dial-up connection.

It took approximately 45 minutes to one hour to complete the session.

\subsection{Participants}

Twenty-two people participated in the study. Sixteen were recruited from a temporary employment agency and were paid for their services; six were internal employees who volunteered for the study. All participants were native speakers of American English and none had worked in the area of speech recognition or natural language understanding. There were eleven males and eleven females. Participants were asked to give their ages in terms of five age categories. The youngest participants were in the 18-20 year-old category and the oldest were in the 51-65 category.

\subsection{Data Collection}

An audio recording which contained both the human participant's utterances and the system's responses was made for each session. In addition, a log file was generated for each session which contained what the system recognized for each utterance as well as the 
system's response. The transcript of the audio recording and the computer-generated log were merged for data analysis.

In addition. participants filled out a questionnaire about their reactions to the system at the end of the session.

\section{RESULTS}

\subsection{Variety of Speaking Styles}

The 22 participants exhibited a considerable variety of speaking styles. A few adopted a telegraphic. command-like style (e.g., "pay telephone bill"). others used a more conversational style (e.g. "I would like to pay my telephone bill"), while others rambled on (see examples in section 4.5). Many made deictic reference (e.g. "pay the bills we just spoke about"). and quite a number used polite forms (e.g. "Please pay...." "Could you tell me...").

The average utterance length was 5.95 words. but ranged from an average of 4.28 for the top-performing quartile of participants (based on the goal success measure described in section 4.3) to 7.98 words for the lowest- performing quartile. This relationship between utterance length and recognition success may be mediated by the greater disfluency rate for longer utterances (Oviatt. 1995) and the tendency for longer utterances to be syntactically more complex.

\subsection{Accuracy}

One measure of accuracy assessed how well the system understood the participant on an utterance-byutterance basis. This measure is based on analysis of three aspects of a user-system interaction: the user's spoken utterance. the words recognized by the system (recognized result), and the system's response. Each spoken utterance was classified into one of three categories: 1. Exact match -- the recognized result exactly matched the speaker's utterance. 2. Exact to user -- the recognized result and speaker's utterance were not an exact match, but the system's response was the same as it would have been for an exact match. Because many different recognized results may be mapped to the same system response. it is possible for the appropriate response to be given to the user. even if the system does not recognize the user's utterance exactly. For example. consider the following interaction:
User: The balance of my checking account.

System recognition: about my checking balance. System response: Your checking balance is $\$ 70.00$.

3. Error -- the system did not give the user the appropriate response.

Thirty-eight percent of the utterances fell into the exact match category. $20 \%$ were in the exact to user category. and $42 \%$ were errors. Thus, the average effective utterance accuracy rate (exact + exact to user) experienced by the participants was $58 \%$. This number had a wide range, from $16 \%$ to $82 \%$ across the 22 participants.

The percentage of utterances in each category as a function of scenario is shown in Figure 1. This graph shows a tendency for the percentage of exact matches to increase and the percentage of errors to decrease as users gain more experience with the system. The outlier point at scenario 5 is due to a large number of competence errors (see section 4.4) in which participants asked the system a question it was not designed to answer.

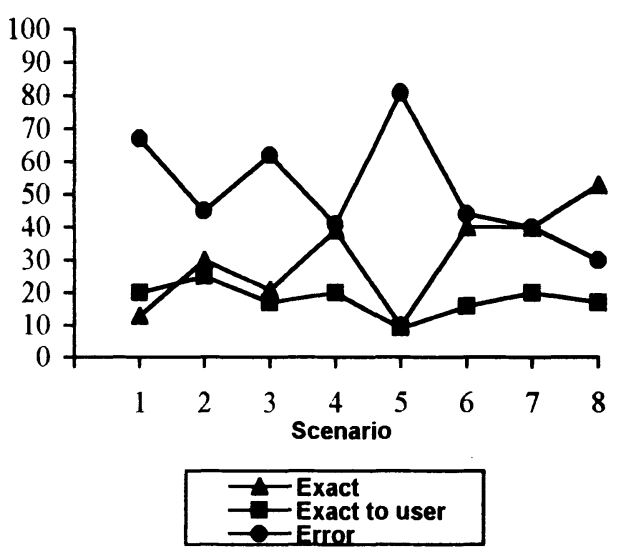

Figure 1. Percentage in each Accuracy category as a function of scenario

Perceived accuracy is likely to be a contributor to overall satisfaction. so it is interesting to compare perceived and actual accuracy. Figure 2 shows participants estimate of the percentage of time the system correctly understood their request and the estimate of the percentage of time it misunderstood their request (collected in independent questions), as well as effective percentage correct (exact + exact to 
user) and error percentage. This graph reveals that study participants tended to overestimate the accuracy of the system and. correspondingly, underestimate the error rate. Participants perceived and actual error rates were significantly correlated $(r=.43, p<.05)$. but perceived and actual percentage correct were not significantly correlated.

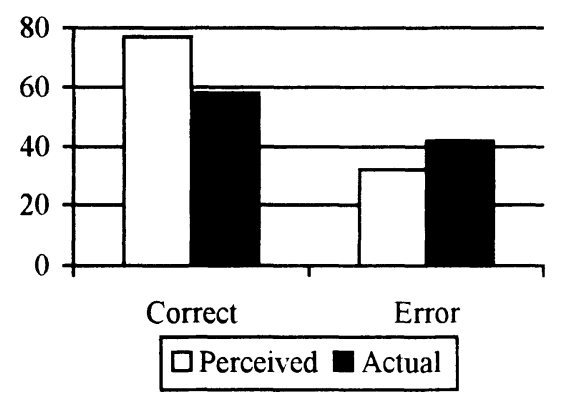

Figure 2. Perceived and Actual Accuracy

\subsection{Goal Success}

Another measure of the effectiveness of the system is whether or not participants were able to accomplish their goals using the it. Almost all of the scenarios required users to perform a number of sub-goals. such as getting the checking balance. paying the phone bill. We scored whether or not a participant was able to accomplish each sub-goal attempted. regardless of the number of consecutive attempts it took. Participants were able to accomplish $92 \%$ of the sub-goals attempted. This number ranged from $75 \%$ to $100 \%$ for the 22 participants. Thus. although accuracy as measured on an utterance-by utterance basis was $58 \%$. the goal success rate was considerably higher. As might be expected. the goal success measure and effective accuracy were highly correlated ( $r=81$. $\mathrm{p}<.05$ ).

\subsection{Error Categories}

Errors were classified into a number of categories as shown in Figure 3. The largest proportion of errors (26\%) were misrecognition errors, in which the system recognized the user's utterance as a different, legitimate request. This includes cases in which the system responded with the required information or action but gave unrequested information as well. cases in which the system was "on the right track" but gave only a partial response. as well as outright errors. About $20 \%$ of the errors were recognition failures. in which the system was unable to produce a match for the user's utterance. For both these categories, the problem resulted from an error of speech recognition and the utterance would have been handled correctly by the natural language component.

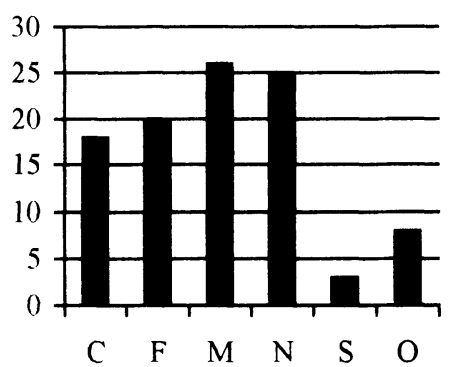

Figure 3. Percentage of Errors due to Competence $(\mathrm{C})$. Recognition Failure (F). Misrecognition (M). Noise (N). Sub-dialog (S) and Other (O)

About $25 \%$ of the errors were due to noise. in which the system picked up background noise or breathing noises when nothing was spoken. or speech intended for the experimenter. These errors are due to the combined effects of the sensitivity of the telephone microphone to background sounds. the speech recognizer's ability to distinguish signal from noise. and the fact that the system as tested could not be put on hold or mute.

About $18 \%$ of the errors were competence errors, in which the user made a request which was outside the system 's competence. For example. one user made the request. "If I write a check for $\$ 200$. will it bounce?" Some of the requests in this category were for information or actions which the system could handle if phrased in a different way, such as this example. Other requests were beyond the system's capabilities. such as providing a history of bill payments.

Sub-dialog errors were errors in which the participant was in a sub-dialog but made a top level dialog request. In many of these cases, the participant had gotten into the sub-dialog as a result of an earlier recognition error and essentially repeated the earlier request. 


\subsection{How People Respond to Errors}

As described in section 4.4. the major sources of error were speech recognition errors. noise. competence errors, and sub-dialog errors. The problem for users is that they often have little idea why the error occurred and what they can do to recover from it. This is related to the what-can-I-say problem (Yankelovich, 1996). As a starting point in designing user interfaces that help people recover from errors, we did a qualitative analysis of the strategies participants used in dealing with errors. We have not yet done a rigorous validation of the classification or a tally of the error strategies. but present this information because of its value in stimulating thinking about how to facilitate error recovery.

Repetition - Often people were at a loss to rephrase their request in a different way and simply repeated the request. This never worked in the case of competence errors. but occasionally worked for recognition errors.

Semantic/syntactic substitution - Participants rephrased a request by making semantic or syntactic substitutions (or both). The first example illustrates a semantic change with a minor syntactic change: the second illustrates a larger syntactic change. Our impression is that rephrasings that involved larger changes tended to be more successful.

Original: Uhm. what is my current insurance bill?

Follow-up: What is the balance on my insurance?

Original: I would like to know my checking balance.

Follow-up: How much is in my checking account?

Elaboration - Participants elaborated on a failed request, adding redundant or new information. In the few cases where a very brief original request was expanded, this strategy worked. Although elaboration often works with human conversation partners. in most cases. the elaboration strategy produced a long request which failed. The following example comes from the participant with the highest utterance error rate.

Original: Uhm. how much would my electric company bill be this month?

Follow-up (1): My electric bill for the month. uh. just wondering how much would come out to this month?
Follow-up (2): My electricity bill. I want to know, uhm. what the amount will be at the end of the month, how much will I owe?

Simplification - Participants simplified a request. resulting in a briefer request. This sometimes also involved breaking a compound request into parts. This strategy often worked. For example.

Original: I would like to take care of my bills. and I would like to know the checking account balance.

Follow-up: What is my checking account balance?

Ignore sub-dialog prompt - When participants found themselves in a sub-dialog due to a recognition error. they sometimes ignored the prompt because it did not offer them acceptable alternatives. Because the system was designed to handle responses to the prompt only, this strategy failed. For example.

Original: I want a transfer...

System: Which bills should I pay?

Follow-up (1): I want a transfer. of a hundred dollars...

System: Which bill? Phone. insurance, Mastercard. or electricity?

Follow-up (2): No. no bills \{pause\}. I just want to transfer money from my savings account into the checking...

Goal shift - On occasion. participants changed their goal when faced with an error. There are at least three situations when this happened: 1. The system responded with information or began a transaction that was relevant to the scenario (although not the correct response to the user's request). and the participant changed his/her goal to match the response. 2. The participant had attempted a request that was beyond the application's competence (such as providing bill payment history). and the changed his/her goal to one within the system 's abilities. 3 . The user gave up on the goal and tried something else.

Help request - Although study participants listened to an introductory message at the beginning of the session that told them they could ask for help. and one of the error prompts reminded them of this, there were an average of only 1.7 help requests per participant. Nine participants never used the help command.

\subsection{Subjective Ratings of System and Correlations with Objective Measures}

At the end of the session. we gave participants a questionnaire which asked them to rate the system on a 
number of dimensions using a 6-point rating scale (1 to 6). The means suggest a favorable reaction to the system (see Table 1 for selected means).

\begin{tabular}{|l|l|}
\hline Dimension & Mean \\
\hline Felt in control (not at all-very much) & 4.50 \\
\hline Ease of use (rather difficult-very easy) & 4.41 \\
\hline Keypad-Speech preference & 4.23 \\
\hline
\end{tabular}

Table 1 . Mean ratings $(1-6)$ on selected questions

The Keypad-Speech question asked participants which system they would be more likely to use if both a keypad and speech system like the current one were available. Forty-five percent of the participants responded in the highest category in favor of speech. indicating that a large group of people had a strong preference for the conversational interface.

The control and ease of use dimensions were significantly correlated with the objective goal success measure $(r=.53, r=.46$. respectively. $p<.05)$. The utterance accuracy measure was moderately. but not significantly, correlated with these measures. This result suggests that the ability to accomplish one s goal may be a more important factor in user satisfaction than utterance level accuracy.

\subsection{Personality Ratings}

It has been observed that people have social reactions to computer systems, particularly those that use speech in the user interface (Nass, Steuer and Tauber. 1994). In the questionnaire, we asked participants to rate the system on 12 personality attributes using a 6-point semantic differential scale. Table 2 . which shows a subset of these attributes, indicates that people rated the personality of the system quite positively. Of particular note is that the system was perceived as efficient. competent, and reliable.

\begin{tabular}{|l|c|}
\hline \multicolumn{1}{|c|}{ Attribute } & Mean \\
\hline Friendly-Unfriendly & 2.05 \\
\hline Active-Passive & 3.00 \\
\hline Intelligent-Unintelligent & 2.41 \\
\hline Helpful-Unhelpful & 2.64 \\
\hline Cooperative-Uncooperative & 2.36 \\
\hline Efficient-Inefficient & 2.27 \\
\hline Reliable-Unreliable & 2.00 \\
\hline Competent-Incompetent & 1.82 \\
\hline
\end{tabular}

Table 2. Mean Personality Ratings (1-6)

\subsection{DISCUSSION AND CONCLUSION}

This study suggests that state-of-the-art conversational interfaces. such as the Conversation Machine. may be preferred over keypad interfaces by typical consumers. The system tested in this study was preferred to a keypad interface by a majority of the participants. It was rated as easy to use. gave the users a sense of control. and was perceived as competent. efficient and reliable. It should be noted that the characteristics of the domain (e.g. number and complexity of potential requests) are critical to the applicability of a conversational interface.

Our results indicate that recognition accuracy may not be the best predictor of user satisfaction. The important subjective measures of ease of use and sense of control were significantly correlated with goal success. but not with utterance accuracy. Goal success was also correlated with both the perceived percentage correct and the perceived percentage error, whereas actual accuracy was correlated with only the perceived error rate, and not the perceived percentage correct. This supports the claim that the user's ability to accomplish his/her goals may be a more important measure of the acceptability of this system (and others like it) than objective accuracy.

The study analyzed categories of errors and participants error recovery strategies as a starting point in improving both goal success and accuracy. Misrecognitions and recognition failures due to the speech recognition component were responsible for the largest proportion of the errors. These errors may be addressed by improvements to the underlying speech technology and also by changes to the user interface which help users avoid and recover from errors. Another significant category of errors was noise. As mentioned above. these errors are due to the sensitivity of the telephone microphone. the speech recognizer's ability to distinguish signal from noise, and the fact that the system tested had no way to mute or pause the recognizer. We have now added a pause function to the Conversation Machine. which gives the user some control over when the system is listening for speech. A third category of errors consisted of competence errors. In some cases, these errors represent the need to add additional functionality to the banking application or additional constructions to the natural language/dialog component. However, there is a tradeoff between increasing the coverage of these components and decreasing the speed and accuracy of 
the system. Furthermore, it is unlikely that a system could be designed to handle some of the utterances produced by our users (see section 4.5). The approach required in these cases is to design the user interface to help people avoid and recover from errors.

Our analysis of participants' strategies in dealing with errors suggests that their intuitions based on human-human conversation did not always work. although some strategies were more likely to be successful than others. The appropriate error recovery strategy may depend on the source of the error. However, the user is not likely to understand the cause of the error and the system may have limited ability to diagnose the cause (or even detect that an error occurred). Consequently, approaches to error recovery must be based on a combination of giving the user generally useful advice, giving the user information sufficient to determine the state of the system. and whatever limited information the system can provide in diagnosing an error. We are currently trying out several approaches to dealing with errors.

Recognition failures are perhaps easier to deal with than other errors since the system has detected that it is in an error state. One approach we are experimenting with is to provide increasingly directive prompts. For example. since simplification tends to be a successful user strategy, one prompt suggests the use of "short, simple requests." If the user is still not successful, examples of acceptable requests are given. The goal is to give users some insight into the style of speech which is acceptable as well as what they can request, without giving the impression that they are constrained to use just the speech given in the examples. Another approach. which can be combined with the above, is to prompt the user to ask for help on a particular topic. See Yankelovich (1996) for a discussion of current wisdom on how to deal with the what-can-I say problem.

Misrecognitions are more difficult to handle, since the system has not detected the error. We are exploring the possibility of using the dialog history as a way to detect repeated misrecognitions.

Errors that occur in a sub-dialog can be addressed in several ways. First. it may be necessary to give the user explicit feedback for state in each prompt, even if that feedback has just been given in the preceding prompt. Second, as our system and many others do. the acceptable responses to the sub-dialog can be presented in a prompt. Finally, there should always be a way for the user to get out of the sub-dialog (e.g. say "cancel"), and the user should be reminded of how to do this.

\subsection{REFERENCES}

Clark. H. H. and Brennan. S. E. (1991). Grounding in communication. In J. Levine, L. B. Resnick, and S. D. Behrand (Eds.), Shared Cognition: Thinking as Social Practice. APA Books. Washington.

Danis, C. and Karat, J. (1995). Technology-driven design of speech recognition systems. In G. Olson and S. Schuon (Eds.) DIS '95: Symposium on Designing Interactive Systems. ACM. New York. 17-24.

Goldberg. A. E. (1995). Constructions: A construction grammar approach to argument structure. The University of Chicago Press. Chicago.

Halstead-Nussloch. R. (1989). The design of phone-based interfaces for consumers. CHI '89 Conference Proceedings, 347-352.

Hansen. B.. Novick. D. G.. and Sutton. S. (1996). Systematic design of spoken prompts. CHI '96 Conference Proceedings, . 157-164.

Marx. M. and Schmandt. C. (1996). MailCall: Message presentation and navigation in a nonvisual environment. CHI '96 Conference Proceedings. 165172.

Nass. C.. Steuer, J. and Tauber. E. R. (1994). Computers are social actors. CHI ' 94 Conference Proceedings. 72-78.

Oriatt. S. Predicting spoken disfluencies during human-computer interaction. (1995). Computer Speech and Language, 9. 19-35.

Rhyne. J. R. and Wolf. C. G. (1993). Recognition based user interfaces. In R. Hartson and D. Hix (Eds.), Advances in Human-Computer Interaction, 4. Ablex. 191-250.

Yankelovich. N. (1996). How do users know what to say? interactions. 3.6. 32-43.

Yankelovich, N., Levow, G. A., and Marx, M. (1995). Designing SpeechActs: Issues in speech user interfaces. CHI '95 Conference Proceedings, 369-376.

Zadrozny, W. (1995). From utterances to situations: Parsing with constructions in small domains. Language, Logic and Computation: The 1994 Moraga Proceedings.

Zadrozny, W. (1996) Natural language processing: Structure and complexity. Proceedings of the Eight International Conference on Software Engineering and Knowledge Engineering, 595-602. 